\title{
Quantitative trait loci for a neurocranium deformity, lack of operculum, in gilthead seabream (Sparus aurata L.)
}

\author{
D. Negríın-Báez, A. Navarro, J. M. Afonso, M. A. Toroand M. J. Zamorano*
}

\section{Summary}

\begin{abstract}
Lack of operculum, a neurocranial deformity, is the most common external abnormality to be found among industrially produced gilthead seabream (Sparus aurata L.), and this entails significant financial losses. This study conducts, for the first time in this species, a quantitative trait loci (QTL) analysis of the lack of operculum. A total of 142 individuals from a paternal half-sibling family (six full-sibling families) were selected for QTL mapping. They had previously shown a highly significant association with the prevalence of lack of operculum in a segregation analysis. All the fish were genotyped for 106 microsatellite markers using a set of multiplex PCRs (ReMsa1-ReMsa13). A linear regression methodology was used for the QTL analysis. Four QTL were detected for this deformity, two of which (QTLOP1 and QTLOP2) were significant. They were located at LG (linkage group) nine and LG10 respectively. Both QTL showed a large effect (about 27\%), and furthermore, the association between lack of operculum and sire allelic segregation observed was statistically significant in the QTLOP1 analysis. These results represent a significant step towards including marker-assisted selection for this deformity in genetic breeding programmes to reduce the incidence of the deformity in the species.
\end{abstract}

Keywords linear regression, microsatellite, opercular complex, quantitative trait loci
Lack of operculum can affect a high proportion of the aquaculture output of gilthead seabream (Sparus aurata L.) (Prestinicola et al. 2013). Although this abnormality does not directly affect growth traits and fish quality, it has been related with a lower resistance to environmental stress in the fish and a higher predisposition towards bacterial infection of the gills (Prestinicola et al. 2013). Furthermore, lack of operculum affects the final appearance of the product and reduces its commercial value, so hatcheries have to sort the fish manually to select and eliminate the fish with this deformity. Despite the strategy, several fish show this deformity at commercial size (Negrín-Báez et al. 2015b). Many studies determined that the prevalence of this deformity can be reduced, but not eliminated, by controlling environmental factors (Prestinicola et al. 2013). Other studies suggest not only that the environment at an early age is the main factor influencing the prevalence of lack of operculum but also that it shows low additive genetic estimation when the fish reach a larger size (Lee-Montero et al. 2015). Marker-assisted selection (MAS) implemented with the identification of the genomic regions responsible for phenotypic variations [quantitative trait loci (QTL)], when included in breeding programmes, could be a useful tool to reduce the prevalence of lack of operculum. It is especially pertinent for traits that are difficult to measure, for ones measured later in development and for those showing a low heritability (Yue 2014), such as lack of operculum in gilthead seabream (Castro et al. 2008; Lee-Montero et al. 2015). Hence, microsatellite markers are useful molecular markers for genetic mapping, and they can be used in multiplex PCR to reduce costs and minimise errors in QTL search processes (Negrín-Báez et al. 2015a). QTL for commercially interesting traits have been reported in gilthead seabream using microsatellite markers: QTL related to growth, sex determination and several morphometric traits were found by Loukovitis et al. (2011, 2012, 2013), two QTL for resistance to pasteurellosis were found by Massault et al. (2010), and one significant QTL for morphometric traits and two suggestive QTL for stress response to confinement were detected by Boulton et al. (2011). However, no QTL for operculum deformity has been reported.

In this study, mating structure, rearing conditions, phenotypic assessment and parental assignment were 
previously described in detail by Negrín-Báez et al. (2015b). Offspring from an industrial broodstock from the PROGENSA ${ }^{\circledR}$ breeding programme were reared to 689 days post-hatching $(524.4 \pm 12.6 \mathrm{~g})$. A sample of 810 fish was phenotypically analysed to identify vertebral deformities and/or lack of operculum. Fish not showing any of these deformities were considered normal. Pedigrees were determined by genetic characterisation, specifically using the microsatellite multiplex PCR SMsa1 (SuperMultiplex Sparus aurata) (Lee-Montero et al. 2013). Sixty-six full-sibling families were formed, and the prevalence of lack of operculum was the only deformity showing a statistically significant association with any breeder or family. About $50 \%$ of individuals suffering from lack of operculum were descendants of one sire (called $\sigma^{\prime} 1_{O}$ ). Therefore, descendants of this sire (six full-sibling families, FAM6 to FAM11) were selected for QTL mapping, and their phenotypes were coded as normal (1) or lack of operculum (2). All the individuals analysed per family and their phenotypes are shown in Table 1. Genotyping was performed using 13 multiplex PCRs (ReMsa1-ReMsa13), previously described by Negrín-Báez et al. (2015a). These multiplex PCRs include 106 microsatellite markers located on the genetic map of this species (Franch et al. 2006). A linear regression (Knott et al. 1996) was performed using GRIDQTL software (Seaton et al. 2006). Half-sibling and fullsibling regression analyses were carried out. Chromosomewide and genome-wide significance thresholds were estimated at $P=0.05$ and $P=0.01$ with a permutation test set to 10000 iterations (Churchill \& Doerge 1994). A QTL was considered suggestive when its significance was between $5 \%$ and $1 \%$ and was considered significant when it was below $1 \%$ at the chromosome-wide level. It was considered significant when significance was between 5\% and $1 \%$ at the genome-wide level (Rodríguez-Ramilo et al. 2011; Vallejo et al. 2014). Family was included as a fixed factor in the model for half-sibling analysis. Confidence intervals were calculated by bootstrapping the samples 10000 times. The effect of the QTL in terms of the percentage of variance explained (PVE) was estimated using the following models, pursuant to Knott et al. (1996):

Table 1 Family structure, number of analysed offspring and number of fish showing each phenotype.

\begin{tabular}{|c|c|c|c|c|c|}
\hline Sires & Dams & Families & $n$ & $\begin{array}{l}\text { Lack of } \\
\text { operculum }\end{array}$ & Normal \\
\hline \multirow[t]{6}{*}{ ○10 } & $\$ 10$ & FAM6 & 13 & 6 & 7 \\
\hline & $\$ 2 O$ & FAM7 & 5 & 1 & 4 \\
\hline & 930 & FAM8 & 79 & 10 & 69 \\
\hline & $94_{O}$ & FAM9 & 10 & 1 & 9 \\
\hline & $95 \circ$ & FAM10 & 6 & 1 & 5 \\
\hline & 960 & FAM11 & 29 & 4 & 24 \\
\hline Total & & & 142 & 23 & 118 \\
\hline
\end{tabular}

$n$, number of analysed offspring in each family.

$$
\begin{aligned}
& \operatorname{PVE}_{\mathrm{HS}}(\%)=4 \times((\text { RMS red }- \text { RMS full }) / \text { RMS red }) \times 100 \\
& \operatorname{PVE}_{\mathrm{FS}}(\%)=2 \times((\text { RMS red }-\mathrm{RMS} \text { full }) / \mathrm{RMS} \text { red }) \times 100,
\end{aligned}
$$

where HS is the half-sibling analysis, FS is the full-sibling analysis, RMS red is the residual mean square from the reduced model in which the QTL effect is excluded and RMS full is the residual mean square from the model in which the QTL effect is fitted. The classification of PVE magnitude was established as a small effect when it was lower than 5\% and a large effect when it was higher than $10 \%$ (Massault et al. 2011).

The association between phenotype and sire allele segregated for microsatellite markers that were close to each significant QTL was determined by contingency tables and Pearson chi-square tests. A significant association was considered when $P \leq 0.05$.

A total of 106 microsatellite markers were genotyped, five of which were non-informative markers because either the breeders were homozygous or null alleles were observed. The length of the gilthead seabream genetic linkage map is $1241.9 \mathrm{cM}$, and $916.9 \mathrm{cM}$ was covered in this study (73.8\%). The covered length was significantly higher than that in other QTL studies in this species (Boulton et al. 2011; Loukovitis et al. 2012). There was an average of four microsatellite markers per linkage group, and the average distance between microsatellite markers was $13.8 \mathrm{cM}$, which is shorter than the recommended maximum distance for QTL searching (20 cM) (Massault et al. 2008).

With respect to QTL related to the lack of operculum deformity, two significant QTL (QTLOP1 and QTLOP2) and two suggestive QTL (QTLOP3 and QTLOP4) were detected at the chromosome-wide level using half-sibling analysis. QTLOP1 was also significant at the genome-wide level, a more stringent statistical level (Loukovitis et al. 2012), and it was also significant in the full-sibling analysis. Thus, QTLOP1 seems to be the most robust QTL for this deformity (Table 2). Both significant QTL had significant effects (more than $25 \%$ of the phenotypic variance). However, these values were estimated within a single half-sibling family, so they should be verified in other families. Their 95\% confidence intervals were very large, so new microsatellite markers with genetic variability for this male in these LGs would be necessary to obtain more exact QTL positions. In this regard, there are other microsatellite markers (seven at LG9 and three at LG10) in the genetic linkage map of gilthead seabream (Franch et al. 2006; Massault et al. 2010), which could be used to improve the QTL mapping, which were not included in the set of multiplexes.

The informative close-to-QTLOP1 microsatellite marker was $G d-78-F(40.5 \mathrm{cM})$, which showed a significant association $(P$-value $=0.02)$ between sire allelic segregation and presence of the deformity in offspring. No significant association with any closely located markers was found with respect to QTLOP2. 
Table 2 Description of QTL detected for lack of operculum deformity in gilthead seabream from full-sibling (FS) and paternal half-sibling (pHS) with full-sibling as fixed-effect analyses, linkage group (LG), position in $\mathrm{CM}, 95 \%$ confidence interval in $\mathrm{CM}(\mathrm{Cl})$, statistical value for QTL (F) and percentage of variance explained (PVE).

\begin{tabular}{|c|c|c|c|c|}
\hline \multirow[b]{2}{*}{ QTL } & \multirow[b]{2}{*}{ LG } & & \multicolumn{2}{|l|}{ Analysis } \\
\hline & & & FS & $\mathrm{pHS}$ \\
\hline \multirow[t]{3}{*}{ QTLOP1 } & 9 & Position $(\mathrm{Cl})$ & $29(21-49)$ & $19(2-88)$ \\
\hline & & $\mathrm{F}$ & $33.1^{2}$ & $11.6^{2,3}$ \\
\hline & & PVE & 7.4 & 28.9 \\
\hline \multirow[t]{3}{*}{ QTLOP2 } & 10 & Position (Cl) & & $31(16-46)$ \\
\hline & & $\mathrm{F}$ & & $10.2^{2}$ \\
\hline & & PVE & & 25.1 \\
\hline \multirow[t]{3}{*}{ QTLOP3 } & 12 & Position (Cl) & & $88(48-93)$ \\
\hline & & $\mathrm{F}$ & & $6.4^{1}$ \\
\hline & & PVE & & 15.2 \\
\hline \multirow[t]{3}{*}{ QTLOP4 } & 14 & Position $(\mathrm{Cl})$ & & $84(3-96)$ \\
\hline & & $\mathrm{F}$ & & $7.2^{1}$ \\
\hline & & PVE & & 17.5 \\
\hline
\end{tabular}

Position $(\mathrm{Cl})$ in $\mathrm{CM}, 95 \%$ confidence interval in $\mathrm{cM}$; $\mathrm{F}$, statistical value for QTL.

${ }^{1} P \leq 0.05$ at chromosome level (suggestive $\mathrm{QTL}$ ).

${ }^{2} P \leq 0.01$ at chromosome level (significant $Q T L$ ).

${ }^{3} P \leq 0.05$ at genome level (significant $\mathrm{QTL}$ ).

These QTL for lack of operculum in gilthead seabream, QTLOP1 and QTLOP2, highlight the possibility of considering this trait in MAS-implemented breeding programmes for this species, especially QTLOP1, as it could be a simple way to reduce the incidence of the most common external deformity and, thus, the financial losses it entails in the gilthead seabream industry. However, these results should be verified in other gilthead seabream families before they are used for genetic selection.

\section{Acknowledgements}

This study was supported by the 'Search of Deformities QTL in Gilthead Seabream' research project (Plan Nacional de $\mathrm{I}+\mathrm{D}+\mathrm{i}, \quad$ AGL2008-04842), PROGENSA ${ }^{\circledR}$ project (Spanish Ministry of Environment, Rural and Marine, JACUMAR). Davinia Negrín-Báez was supported by a grant from the Cabildo Insular de Gran Canaria. We are grateful to Guacimara Alejandro (MINECO, PTA2012-8105-I) and Ivonne Lee-Montero for their technical support, and to Silvia Rodríguez-Ramilo for her help and advice.

\section{References}

Boulton K., Tsigenopoulos C.S., Massault C., Houston R.D., de Koning D.J., Haley C.S., Bovenhuis H., Batargias C., Canario A.V.M. \& Kotoulas G. (2011) QTL affecting morphometric traits and stress response in the gilthead seabream (Sparus aurata L). Aquaculture 319, 58-66.

Castro J., Pino-Querido A., Hermida M., Chavarrías D., Romero R., García-Cortés L.A., Toro M.A. \& Martínez P. (2008) Heritability of skeleton abnormalities (lordosis, lack of operculum) in gilthead seabream (Sparus aurata) supported by microsatellite family data. Aquaculture 279, 18-22.

Churchill G.A. \& Doerge R.W. (1994) Empirical threshold values for quantitative trait mapping. Genetics 138, 963-71.

Franch R., Louro B., Tsalavouta M. et al. (2006) A genetic linkage map of the hermaphrodite teleost fish Sparus aurata L. Genetics 174, 851-61.

Knott S.A., Elsen J.M. \& Haley C.S. (1996) Methods for multiplemarker mapping of quantitative trait loci in half-sib populations. Theoretical and Applied Genetics 93, 71-80.

Lee-Montero I., Navarro A., Borrell Y. et al. (2013) Development of the first standardized panel of two new microsatellites multiplex PCRs for gilthead seabream (Sparus aurata L.). Animal Genetics 44, 533-46.

Lee-Montero I., Navarro A., Negrín-Báez D. et al. (2015) Genetic parameters and $\mathrm{G} \times \mathrm{E}$ interactions for skeleton deformities and growth traits at different ages on gilthead seabream (Sparus aurata L.) in four Spanish regions. Animal Genetics, 46, 164-74.

Loukovitis D., Sarropoulou E., Tsigenopoulos C.S., Batargias C., Magoulas A., Apostolidis A.P., Chatziplis D. \& Kotoulas G. (2011) Quantitative trait loci involved in sex determination and body growth in the gilthead sea bream (Sparus aurata L.) through targeted genome scan. PLoS One 6, e16599.

Loukovitis D., Sarropoulou E., Batargias C., Apostolidis A., Kotoulas G., Tsigenopoulos C. \& Chatziplis D. (2012) Quantitative trait loci for body growth and sex determination in the hermaphrodite teleost fish Sparus aurata L. Animal Genetics 43, 753-9.

Loukovitis D., Batargias C., Sarropoulou E., Apostolidis A.P., Kotoulas G., Magoulas A., Tsigenopoulos C.S. \& Chatziplis D. (2013) Quantitative trait loci affecting morphology traits in gilthead seabream (Sparus aurata L.). Animal Genetics 44, 480-3.

Massault C., Bovenhuis H., Haley C.S. \& Koning D.J. (2008) QTL mapping designs for aquaculture. Aquaculture 285, 1-4.

Massault C., Hellemans B., Louro B., Batargias C., Van Houdt J.K.J., Canario A., Volckaert F.A.M., Bovenhuis H., Haley C. \& de Koning D.J. (2010) QTL for body weight, morphometric traits and stress response in European sea bass Dicentrarchus labrax. Animal Genetics 41, 337-45.

Massault C., Franch R., Haley C., De Koning D.J., Bovenhuis H., Pellizzari C., Patarnello T. \& Bargelloni L. (2011) Quantitative trait loci for resistance to fish pasteurellosis in gilthead sea bream (Sparus aurata). Animal Genetics 42, 191-203.

Negrín-Báez D., Navarro A., Lee-Montero I., Afonso J.M., Sánchez J.J., Elalfy I.S., Manchado M., Sánchez J.A., García-Celdrán M. \& Zamorano M.J. (2015a) A set of 13 multiplex PCRs of specific microsatellite markers as a tool for QTL detection in gilthead seabream (Sparus aurata L.). Aquaculture Research 46(Suppl. 1), 45-5.

Negrín-Báez D., Navarro A., Lee-Montero I., Soula M., Afonso J.M. \& Zamorano M.J. (2015b) Inheritance of skeletal deformities in the gilthead seabream (Sparus aurata) - lack of operculum, lordosis, vertebral fusion and LSK complex. Journal of Animal Science 93, 53-61.

Prestinicola L., Boglione C., Makridis P., Spanò A., Rimatori V., Palamara E., Scardi M. \& Cataudella S. (2013) Environmental conditioning of skeletal anomalies typology and frequency in gilthead seabream (Sparus aurata L., 1758) juveniles. PLoS One 8, e55736. 
Rodríguez-Ramilo S.T., Toro M.A., Bouza C., Hermida M., Pardo B.G., Cabaleiro S., Martínez P. \& Fernández J. (2011) QTL detection for Aeromonas salmonicida resistance related traits in turbot (Scophthalmus maximus). BMC genomics 12, 541.

Seaton G., Hernandez J., Grunchec J.A., White I., Allen J., de Koning D.J., Wei W., Berry D., Haley C. \& Knott S. (2006) GRIDQTL: A grid portal for QTL mapping of compute intensive datasets. In: Proceedings of the 8th World Congress on Genetics
Applied to Livestock Production. August 13-18, Belo Horizonte, MG, Brasil.

Vallejo R.L., Palti Y., Liu S., Evenhuis J.P., Gao G., Rexroad C.E. III \& Wiens G.D. (2014) Detection of QTL in rainbow trout affecting survival when challenged with Flavobacterium psychrophilum. Marine Biotechnology 16, 349-60.

Yue G.H. (2014) Recent advances of genome mapping and markerassisted selection in aquaculture. Fish and Fisheries 15, 376-96. 